\title{
Deteç̧ão automática do espaçamento médio de meios periódicos por sinais ultrassônicos retroespalhados
}

\author{
Adriana Kauati*, Wagner Coelho de Albuquerque Pereira, Marcello Luiz Rodrigues de Campos
}

\begin{abstract}
Resumo A interpretação da imagem ultrassônica, por ocorrer de modo visual e qualitativa, traz uma variação inter e intra-observador importante. A adoção de métodos quantitativos é uma forma de diminuir esta dependência. Entre tais métodos está a quantificação do espaçamento médio entre espalhadores (Mean Scatterer Spacing-MSS), que pode ser útil para detectar mudanças na microestrutura quasi-periódica de tecidos como o hepático ou o esplênico. Neste trabalho foram avaliados três métodos clássicos de estimação espectral para cálculo do MSS (sem intervenção do operador): BURG, WIENER e MUSIC. O intuito é comparar suas potencialidades para a estimação automática de espaçamento médio de espalhadores ultrassônicos. Inicialmente as avaliações foram realizadas com 10.000 sinais simulados a partir de um modelo em que se tem controle das variáveis de interesse, e em seguida foram utilizados sinais reais de phantoms de fios de nylon imersos em água. O método de BURG não conseguiu estimar adequadamente o espaçamento em sinais de phantom, tendo apresentado resultados equivalentes aos outros métodos deste trabalho somente para sinais simulados. $\mathrm{O}$ método de WIENER para os sinais simulados apresentou resultados de menor percentual de acerto, ficando em segundo lugar, para os sinais dos phantoms. O método de subespaço MUSIC apresentou melhor desempenho global em relação a BURG e WIENER, com resultados de $100 \%$ de acerto para o phantom de fio de nylon de $1,2 \mathrm{~mm}$ e $91,45 \%$ para $0,8 \mathrm{~mm}$ considerando uma janela de acerto de $10 \%$.
\end{abstract}

Palavras-chave Espaçamento médio, Espalhadores ultrassônicos, Estimação espectral, Meios periódicos.

\section{Automatic detection algorithms for mean scatterer space estimation using ultrasound backscattered signals}

\begin{abstract}
The interpretation of ultrasound imaging is essentially visual and qualitative, so there are important inter and intra-observer variations. Quantification methods aim at decreasing this dependency. Among those, the quantification of the Mean Scatterer Spacing (MSS) can be useful to detect changes in the microstructure of quasi-periodic tissues, such as liver or spleen. This study evaluated the following methods of spectral estimation for calculating the MSS (without requiring operator intervention): BURG, WIENER and MUSIC. The aim is to compare their potential for automatic estimation of MSS from ultrasonic scattering signals. Initially, the evaluation has been carried out using 10,000 simulated signals, with the aim of studying the behavior of the methods using a model in which the variables of interest can be controlled. Then, the methods have been applied to real signals of nylon phantoms immersed in water. The BURG method could not estimate the spacing of US phantom signals, presenting results similar to the other methods only for simulated signals. The WIENER method for the simulated signals was in second place in terms of percentage of success, when considering signals from the phantoms. The subspace method MUSIC had the best performance from all three methods.
\end{abstract}

Keywords Average spacing, Ultrasonic scatterers, Spectral estimation, Periodic media. 


\section{Extended Abstract}

\section{Introduction}

Technologies based on ultrasound (US) imaging have been increasingly applied in the healthcare area. However, image interpretation depends on visual and qualitative analysis and so is highly operator-dependent. To reduce this dependency, several signal processing techniques have been proposed to quantify characteristics of the internal structures of biological tissues from the original signals of radiofrequency $(R F)$.

Some tissue properties (attenuation coefficient and backscatter, backscattered density, mean diameter particle and Mean Scatterer Spacing (MSS)) have been studied by various researchers: Huang et al. (2008), Insana et al. (1991), Landini and Verrazzani (1990), Machado et al. (2006), Narayanan et al. (1997) and Ossant et al. (2001). The basic idea in all these works is that the variation of the parameter values can be applied in the differentiation between normal structures and pathological, even when it is not apparent in the conventional US.

This work is dedicated to the study the MSS estimation from ultrasonic backscattered waves, which characterizes the spatial organization, and it is of particular interest to detect changes in the microstructure of quasi-periodic tissues as the liver or spleen. This parameter has been calculated by common techniques such as temporal autocorrelation, power spectrum, cepstrum and higher order statistics (Fellingham and Sommer, 1984; Narayanan et al., 1997; Wear et al., 1993). Other researchers have proposed new techniques such as Varghese and Donohue (1994), based on the spectral autocorrelation and Simon et al. (1997), who proposed an algorithm for MSS estimation based on spectral redundancy generated by a quadratic transformation of ultrasonic RF signals.

This paper presents two objectives: to evaluate three of the best known modern parametric methods of spectral estimation, $B U R G$, WIENER and MUSIC, applied to estimate the MSS of periodic medium and propose an automatic method of determining the order of the model.

\section{Material and Methods}

The study is initially performed on simulated ultrasound (US) signals based on a linear model of the US interaction with biological tissue following the model used by Pereira and Maciel (2001). The evaluation of the algorithms was performed using 10,000 simulated signals with different levels of Ad (regular part/diffuse) and jitter (variation in the expected position of the particle regular), divided into 20 groups of 500 signals each. The sampling frequency is $25 \mathrm{MHz}$, the transducer central frequency is $3.5 \mathrm{MHz}$ with a bandwidth of $1.5 \mathrm{MHz}$.

Other analyzed signals were from two phantoms of nylon, each built with four wires aligned in the same plane and spaced regularly: one with $1.2 \mathrm{~mm}$ and other with $0.8 \mathrm{~mm}$ (wire diameter $0.5 \mathrm{~mm}$ ) according to Pereira et al. (2002). The phantoms were immersed in water and the RF signals were collected with a transducer (Panametrics) with center frequency and bandwidth of $20 \mathrm{MHz}$.

BURG algorithm (Marple, 1987) was tested using the relative entropy as a method of determination the order of the system (proposed by Kauati, 2000). The basic idea is to calculate the entropy for each model order and elect the one where the entropy is minimal. This method was compared with classical methods of least squared error and Akaike (Marple, 1987) using simulated signals, having performed better than the last two. The orders for the WIENER method (Marple, 1987) were determined by the minimum mean square error, for having presented best performance in relation to the results obtained using the methods of Akaike (Marple, 1987) or entropy. The MUSIC method (Multiple Signal Classification) (Marple, 1987) is based on obtaining the eigenvalues and eigenvectors of the covariance matrix.

\section{Results}

Figure 7 presents the results of percentage of success due the jitter for different levels of Ad (different curves presented for a tolerance range of $\pm 5 \%$ around the correct value of frequency spacing on the simulated signals - in this case it is expected $616.00 \mathrm{kHz}$ for the velocity of ultrasound in the medium of $1540 \mathrm{~m} / \mathrm{s}$ ) for the three methods.

For the phantom signals all methods showed the mean estimated frequencies (Table 3) above $0.94 \mathrm{MHz}$, (corresponding to a period shorter than $0.8 \mathrm{~mm}$ ) and above $0.62 \mathrm{MHz}$ (corresponding to a period shorter than a $2 \mathrm{~mm}$ ) as expected, because the US beam is approximately $5^{\circ}$ to $10^{\circ}$ inclinated with respect to the plane formed by the nylon thread, which would lead to a lower estimate than the actual spacing.

\section{Discussion}

Using simulated signals, the three methods produced similar results to Simon (1997) and Pereira and Maciel (2001), having the advantage of not requiring a priori knowledge of the signal, as in the quadratic signal processing (Simon, 1997) and the use of SSA Pereira and Maciel (2001).

Using signals from phantoms, only the MUSIC method showed similar results to the literature Pereira and Maciel (2001). This may be due to the fact that SSA also separates the spaces of periodic signals with aperiodic signals.

\section{Conclusion}

The MUSIC method showed better results, which is an indication that it should be further investigated with a view to future clinical use. 


\section{Introdução}

A imagem ultrassônica têm tido aplicação cada vez mais ampla na área da saúde e tem avançado continuamente, não apenas em qualidade mas principalmente em modos diferentes de processar o sinal de eco para obtenção de novas características nas imagens (harmônicos, inversão de fase, etc). Entretanto, a interpretação da mesma é operador-dependente, pois ocorre de forma visual e qualitativa, trazendo uma variação inter e intra-observador importante (Calas et al., 2010). Visando diminuir esta dependência, os cientistas vem explorando várias técnicas de processamento de sinais para quantificar características das estruturas internas dos tecidos biológicos a partir dos sinais originais de radiofrequência (RF).

Entre as propriedades mais comuns dos tecidos, pode-se citar: coeficientes de atenuação e retroespalhamento, densidade de retroespalhadores, diâmetro médio de partículas e espaçamento médio entre espalhadores (Mean Scatterer Spacing - MSS). Estas propriedades foram estudadas por vários pesquisadores: Bridal et al. (1997), Fellingham e Sommer (1984), Huang et al. (2008), Insana et al. (1991), Landini e Verrazzani (1990), Machado et al. (2006), Narayanan et al. (1997), Ossant et al. (2001), Simon et al. (1997), Varghese e Donohue (1994) e Wear et al. (1993). A hipótese básica assumida por todos esses trabalhos é que a variação nos valores dos parâmetros que quantificam estas propriedades pode ter aplicação na diferenciação entre estruturas normais e patológicas, ainda que não estejam aparentes na ultrassonografia convencional.

Este trabalho se dedica ao estudo da regularidade espacial do meio, quantificada pelo espaçamento médio de espalhadores (MSS - Mean Scatterer Spacing) da onda ultrassônica, que e é de especial interesse para detecção de mudanças na microestrutura quasi-periódica de tecidos como o hepático ou o esplênico. Este parâmetro tem sido calculado por técnicas como: autocorrelação temporal, espectro de potência, cepstrum e estatísticsa de ordem superior (Fellingham e Sommer, 1984; Landini e Verrazzani, 1990; Narayanan et al.,1997; Wear et al., 1993). Varghese e Donohue (1994) tomaram como base a autocorrelação espectral e Simon et al. (1997) e propuseram um algoritmo para estimação de MSS baseado na redundância espectral gerada por uma transformação quadrática dos sinais de RF ultrassônico. Em 2001, Pereira e Maciel aplicaram a técnica de Análise do Espectro Singular (Singular Spectrum Analysis - SSA) para estimar MSS. Já Amin et al. (2005) utilizaram a estimação espectral autorregressiva para estimar a variação de MSS. Todas essas técnicas são aplicadas em sinais de RF, ou em sua envoltória, que contém uma mistura de contribuições de espalhadores periódicos e não-periódicos. Porém, não há na literatura descrição do desempenho de métodos espectrais modernos, e outro ponto importante é que nenhum dos métodos se propõe a ser automático, ou seja, sempre há a interferência do operador para realizar alguma escolha ou estabelecer algum limiar de forma heurística para definir a ordem do modelo.

Nesse contexto, este trabalho apresenta dois objetivos básicos: (a) avaliar três dos mais conhecidos métodos paramétricos modernos de estimação espectral, a saber, BURG, WIENER e MUSIC aplicados à estimação do espaçamento médio de meios periódicos e (b) propor um método automático de determinação da ordem do modelo.

\section{Materiais e Métodos}

O estudo é inicialmente realizado em sinais simulados ultrassônicos, baseado num modelo linear de interação do US com o tecido biológico, para que se tenha maior controle das variáveis envolvidas no processo. Em seguida serão processados sinais reais de phantoms (corpos de prova) compostos de fios de nylon paralelos imersos em água.

\section{Modelo matemático do sinal RF ultrassônico simulado}

Os sinais de RF retroespalhados foram simulados considerando um meio linear e unidimensional, seguindo o modelo utilizado por Pereira e Maciel (2001). Assim, o sinal de eco recebido, em função do tempo, $r(t)$, pode ser escrito como:

$r(t)=p(t) * g(t)+n(t)$

sendo:

- $p(t)=$ pulso de ultrassom transmitido;

- $g(t)=$ função de caracterização do meio (Resposta Impulsiva);

- $n(t)=$ ruído do sistema experimental; e

- * = operação de convolução.

O meio é descrito como contendo uma sequência de partículas espalhadoras distribuídas regularmente, entremeadas por partículas com distribuição aleatória. A função $g(t)$ é definida como o somatório de dois grupos de partículas, um com distribuição espacial periódica e o outro com distribuição aperiódica, conforme Equação 2:

$g(t)=\sum_{i=1}^{N} a_{i} \delta\left(t-\tau_{i}\right)+\sum_{i=1}^{M} b_{i} \delta\left(t-\theta_{i}\right)$

sendo: 
- $\quad N=$ número total de partículas regulares;

- $M=$ número total de partículas difusas;

- $a_{i}=$ amplitude do sinal regular;

- $\tau_{i}=$ atraso (relativo à posição) da parte regular;

- $b_{i}=$ amplitude do sinal difuso;

- $\theta_{i}=$ atraso (relativo à posição) da parte difusa; e

- $\delta$ = função impulso.

Esse modelo é compatível com tecidos biológicos que apresentam estruturas regulares mescladas com difusas como, por exemplo, o tecido hepático, sendo similar aos encontrados na literatura (Simon et al., 1997).

\section{Sinal de RF ultrassônico simulado}

No exemplo da Figura 1, apresenta-se um pulso simulado (domínio do tempo e da frequência), de frequência central 3,5 MHz e largura de banda $1,5 \mathrm{MHz}$, a resposta impulsiva do tecido e o sinal simulado de ultrassom retroespalhado, com espaçamento médio de $1,25 \mathrm{~mm}$ para jitter de $1 \%$ (jitter é definido como a variação na posição da partícula ao redor do seu valor esperado).

\section{Características dos sinais simulados}

Uma primeira avaliação dos algoritmos foi realizada utilizando 10.000 sinais simulados com diversos níveis de Ad - relação sinal da parte regular/sinal da parte difusa - $(11,7 \% ; 41,2 \% ; 58,8 \%$ e $76,5 \%)$ e jitter $(1 \%$, $5 \% 10 \%, 20 \%, 30 \%$ ), divididos em 20 grupos de 500 sinais. A frequência de amostragem é de $25 \mathrm{MHz}$, a frequência central do transdutor é de $3,5 \mathrm{MHz}$ e a largura de banda de frequência é de $1,5 \mathrm{MHz}$.

Os sinais foram simulados com um espaçamento médio entre os espalhadores (MSS) de 1,25 mm (compatível com os tecidos biológicos), cujo valor da frequência correspondente (FreqMSS) é calculado de acordo com a equação a seguir:

$$
\text { FreqMSS }=\frac{c}{(2 x M S S)}
$$

Pulso de sinal

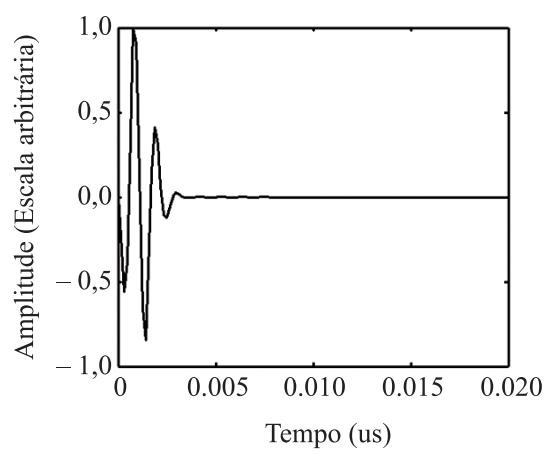

(c)

Resposta impulsiva do tecido

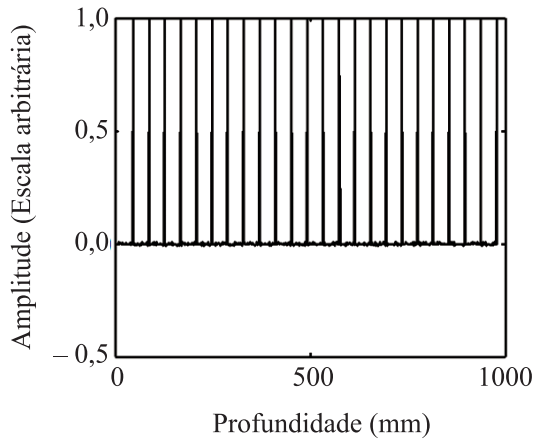

FFT do pulso de US

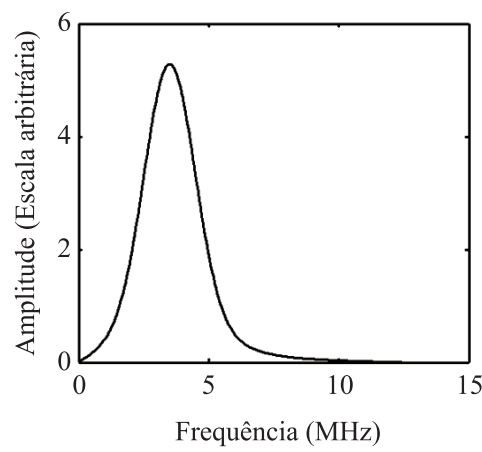

(d)

Sinal simulado

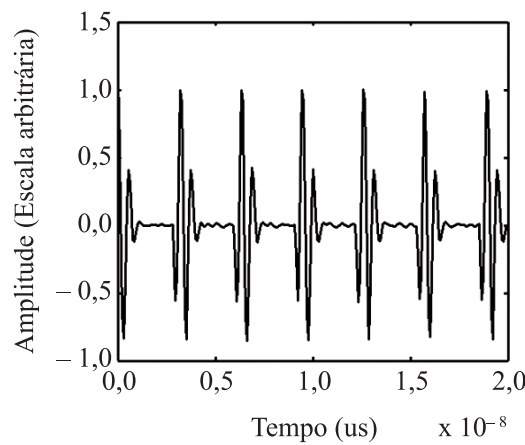

Figura 1. (a) Sinal de excitação simulado de um pulso (frequência central 3,5 MHz) no domínio do tempo e (b) magnitude da FFT do sinal. (c) Resposta impulsiva do tecido e (d) sinal simulado de ultrassom retroespalhado para espaçamento médio de 1,25 mm com jitter de $1 \%$. Figure 1. (a) Signal of a simulated excitation pulse (center frequency $3.5 \mathrm{MHz}$ ) in the time domain and (b) magnitude of the signal FFT. (c) Impulse response of the tissue and (d) simulated signal of backscattering ultrasound for mean spacing of 1.25 mm with a jitter of $1 \%$. 
sendo:

- FreqMSS = frequência de máxima magnitude;

- $c=$ velocidade do ultrassom; e

- $M S S$ = espaçamento médio dos espalhadores.

A Figura 2 apresenta, como exemplo, trechos de sinais simulados. Pode-se observar que a periodicidade fica progressivamente mais difícil de ser identificada visualmente.

\section{Algoritmo de simulação do sinal de $\mathrm{RF}$ ultrassônico}

Baseado no modelo descrito no item anterior, Pereira e Maciel (2001) desenvolveram um algoritmo de simulação de sinais de RF ultrassônicos cujos passos estão descritos a seguir:

1. Definição da frequência central e banda do sinal de excitação (emitido por um transdutor);

2. Definição das características dos espalhadores do meio;

3. Modelo unidimensional, que é equivalente a colocar partículas espalhadoras (regulares e difusas) alinhadas, com uma variação nas suas amplitudes (correspondente à capacidade de reflexão de cada alvo);

4. A distância entre partículas difusas vizinhas segue distribuição uniforme. Já entre partículas regulares vizinhas, a distribuição Gama é a escolhida (Narayanan et al., 1997), cuja equação será descrita mais à frente;

5. As amplitudes das capacidades reflexivas das partes regular e difusa seguem uma distribuição uniforme. São estabelecidos valores máximo e mínimo para as amplitudes da parte regular. Já os valores máximo e mínimo da parte difusa são determinados de forma a atender ao parâmetro Ad (ou relação sinal-ruído), definido a seguir;

6. Cálculo do sinal característico do meio; e

7. A amplitude da parte regular do sinal (coeficientes ai da Equação 2) é calculada como:

$a_{i}=A \min +(A \max -A \min ) \cdot N r_{i}$

sendo:

- $\operatorname{Amin}=$ Amplitude mínima das partículas regulares;

Sinal simulado

(a)

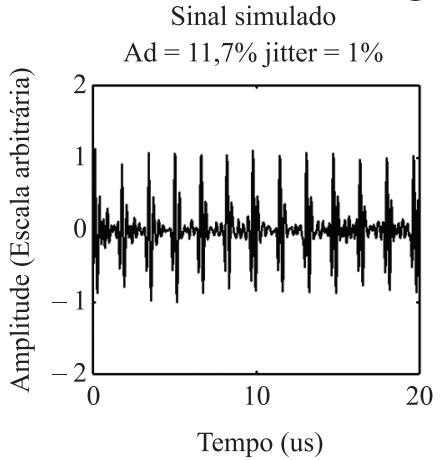

(b)

Sinal simulado
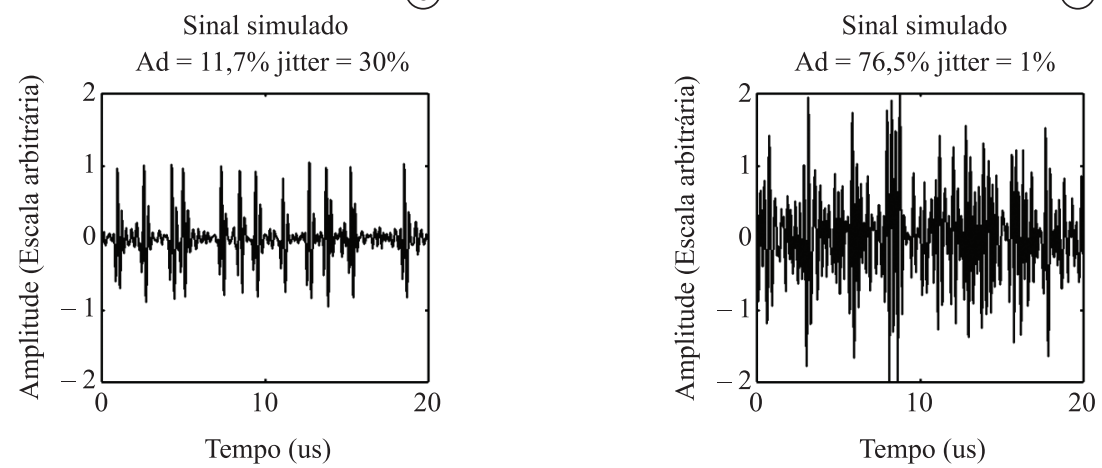

Figura 2. Exemplos de sinais simulados com MSS =1,25 mm: (a) $\mathrm{Ad}=11,7 \%$ e $j i t t e r=1 \%$; (b) e $\mathrm{Ad}=11,7 \%$ e $j i t t e r=30 \% ;$ (c) $\mathrm{Ad}=76,5 \%$ e jitter $=1 \%$

Figure 2. Examples of simulated signals with MSS $=1.25 \mathrm{~mm}:$ (a) $\mathrm{Ad}=11.7 \%$ and jitter $=1 \%$; (b) and Ad $=11.7 \%$ and jitter $=30 \%$; (c) $\mathrm{Ad}=76.5 \%$ and jitter $=1 \%$. 
- $A m a x=$ Amplitude máxima das partículas regulares; e

- $N r_{\mathrm{i}}=$ número randômico com distribuição uniforme.

8. A amplitude parte difusa do sinal (coeficientes $b_{\mathrm{i}}$ da Equação 2) é calculada como o produto:

$b_{i}=2 \cdot A d \cdot\left(N r_{i}-0,5\right)$

sendo:

- $A d=$ razão entre as amplitudes médias dos ecos das partículas difusas e das partículas regulares;

- $N r_{\mathrm{i}}=$ número randômico com distribuição uniforme.

9. A distribuição espacial (unidimensional) da parte regular (coeficientes $\tau_{\mathrm{i}}$ da Equação 2) é do tipo Gama, onde o seu valor médio é o MSS simulado e seu desvio-padrão funciona como uma variação da posição esperada da partícula regular (jitter);

10. A distribuição espacial da parte difusa (coeficientes $\theta_{\mathrm{i}}$ da Equação 2) é uniforme; e

11. Cálculo do pulso de excitação conforme a Equação 6:

$p(t)=-t e^{-4 B_{\omega} t^{2}} \sin \left(\omega_{0} t\right) u(t)$

sendo:

- $\omega_{0}=$ frequência central;

- $B_{\omega}=$ largura de banda; e

- $u(t)=$ função degrau.

Obtenção do sinal de RF ultrassônico, gerado pela convolução do pulso com o meio simulado, podendo ou não se acrescentar ruído branco neste ponto para simular o ruído da instrumentação.
Os valores dos parâmetros de simulação utilizados no presente trabalho se encontram enumerados na Tabela 1.

\section{Phantom de fios de nylon}

Outros sinais analisados foram provenientes de dois phantoms de fios de nylon, cada um construído com quatro fios alinhados num mesmo plano e espaçados regularmente, um deles com $1,2 \mathrm{~mm}$ e outro de $0,8 \mathrm{~mm}$ (diâmetro do fio $0,5 \mathrm{~mm}$ ) conforme Pereira et al. (2002). Os phantoms foram imersos em água e os sinais de RF foram coletados com um transdutor (Panametrics) de frequência central e largura de banda de $20 \mathrm{MHz}$. O transdutor foi posicionado com a face paralela ao comprimento dos fios, executando uma varredura linear em um plano que forma um ângulo aproximadamente de $10^{\circ}-15^{\circ}$ ao plano dos fios para garantir que todos os fios fossem irradiados. Por este motivo, o espaçamento esperado é um pouco menor que $0,8 \mathrm{~mm}$ e $1,2 \mathrm{~mm}$. Os sinais $\mathrm{RF}$ adquiridos foram amplificados e digitalizados a uma taxa de amostragem de $100 \mathrm{MHz}$ em um osciloscópio de 8 bits (LeCroy 9350AL). Foram coletados 152 sinais de cada phantom. Para a velocidade do US na água foi usado o valor padrão a $22{ }^{\circ} \mathrm{C}: 1.498 \mathrm{~m} / \mathrm{s}$.

Trechos de sinal destes phantoms são apresentados na Figura 3. Os sinais foram coletados no Laboratorie d'Imagerie Paramétrique (Université Paris VI, França) e cedidos pelo Prof. Pascal Laugier.

\section{Métodos de análise espectral}

As estimativas espectrais paramétricas possuem as densidades espectrais de potência totalmente definidas pelos termos dos modelos e pela variância do ruído branco do processo. Um dos métodos mais

Tabela 1. Valores dos parâmetros utilizados ecos provenientes do meio simulado.

Table 1. Parameter values using simulated echoes from the medium.

\begin{tabular}{ll}
\multicolumn{1}{c}{ Parâmetro } & \multicolumn{1}{c}{ Valor } \\
\hline Frequência de amostragem & $25 \mathrm{~Hz}$ \\
Frequência de excitação do transdutor & $3,5 \mathrm{MHz}$ \\
Banda do sinal de excitação & $1,5 \mathrm{MHz}$ \\
Velocidade do ultrassom no meio & $1.540 \mathrm{~m} / \mathrm{s}$ \\
Distância média entre os espalhadores regulares & $1,25 \mathrm{~mm}$ \\
Variação percentual das posições dos espalhadores regulares (jitter) $)$ & $0,01(1 \%) \quad 0,05(5 \%)$ \\
Amplitude máxima da capacidade de reflexão das partículas regulares & 1 \\
Amplitude mínima da capacidade de reflexão das partículas regulares & 0,99 \\
Razão entre as amplitudes médias dos ecos das partículas difusas e & $11,7 \%(\cong-18,64 \mathrm{~dB}) 41,2 \%(\cong-7,7 \mathrm{~dB})$ \\
das partículas regulares (Ad) & $57,8 \%(\cong-4,76 \mathrm{~dB}) 76,5 \%(\cong-2,32 \mathrm{~dB})$ \\
Relação sinal/Ruído branco & $0,001 \mathrm{~dB}$ \\
Espessura & $100 \mathrm{~mm}$ \\
Número de partículas difusas & 1000 \\
Número de partículas fixas & Espessura dividida pela distância média entre os \\
\hline
\end{tabular}


Sinal de Phantom Fios de nylon de $0,8 \mathrm{~mm}$

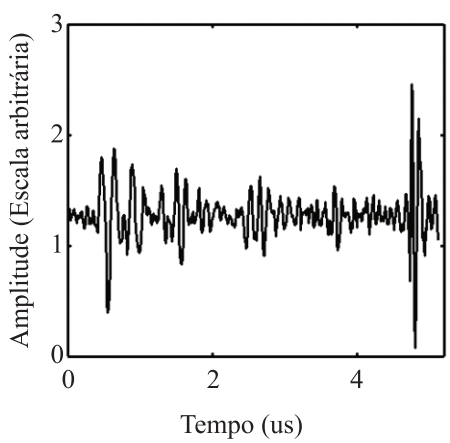

Sinal de Phantom Fios de nylon de $1,2 \mathrm{~mm}$

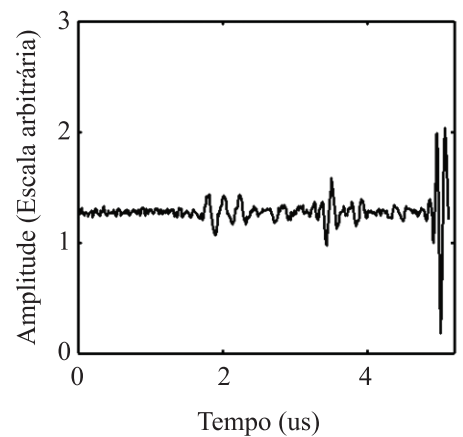

Figura 3. Exemplos de sinais de phantom de fios de nylon espaçados de (a) $0,8 \mathrm{~mm}$ e (b) 1,2 $\mathrm{mm}$.

Figure 3. Signals from nylon phantom spaced by (a) $0.8 \mathrm{~mm}$ and (b) $1.2 \mathrm{~mm}$.

utilizados, segundo Marple (1987), é o autorregressivo (AR), base dos métodos de BURG e WIENER, cujo espectro apresenta picos agudos que estão associados à estimativa espectral de alta resolução. A estimativa dos parâmetros do modelo AR pode ser obtida como solução de uma equação linear e analisada como uma estimativa espectral preditiva linear.

A função de transferência do processo AR para uma entrada discreta e[n] (ruído branco) é dada por:

$$
\begin{aligned}
& H(z)=\frac{1}{A(z)} \\
& A(z)=1+a_{1} z^{-1}+a_{2} z^{-2}+\ldots+a_{M} z^{-M}
\end{aligned}
$$

onde,

- $H(z)=$ a transformada $\mathrm{Z}$ da resposta impulsiva $h[n]$;

- $a_{\mathrm{i}}=$ seus coeficientes; e

- $M=$ ordem.

\section{Método de BURG}

No algoritmo de BURG, também conhecido como algoritmo de máxima entropia (Marple, 1987), os coeficientes da função de transferência $A(z)$ podem ser calculados como:

$$
a_{m}[n]=a_{m-1}[n]+\gamma_{m} a_{m-1}[p-n]
$$

sendo:

$$
\gamma_{m}=\frac{2 \sum_{n=M}^{N-1}\left(e_{m-1}^{f}[n] e_{m-1}^{b}[n-1]\right)}{\sum_{n=M}^{N-1}\left(e_{m-1}^{f}[n]^{2} e_{m-1}^{b}[n-1]^{2}\right)}
$$

- $\quad e_{m}^{f}[n]=$ erro de predição forward;

- $\quad e_{m}^{b}[n]=$ erro de predição backward;

- $M=$ ordem do modelo; e
- $\quad N=$ tamanho do sinal.

Para o método de BURG, foi testado o uso da entropia relativa (Cover e Thomas, 1991) como método de determinação de ordem de sistema, conforme proposto por Kauati (2000). A idéia básica é calcular a entropia para cada ordem do modelo e eleger aquele onde esta é mínima. Este método é comparado com os métodos clássicos de Akaike e mínimo erro quadrático (Marple, 1987).

O tamanho da janela foi definido experimentalmente, utilizando vários tamanhos $\mathrm{e}$ verificando qual obteve melhor desempenho. No caso deste método, as janelas de 200 e de 100 amostras obtiveram desempenho semelhantes, mas devido ao tempo de processamento que o método requer, optou-se por utilizar 100 amostras e overlap de 50 amostras.

Os passos para implementação do método de BURG neste trabalho se resumem da seguinte forma:

I. Define-se o tamanho da janela retangular do sinal;

II. Define-se o overlap para o deslocamento da janela (normalmente $50 \%$ );

III. Para todas as ordens, calculam-se, para todas as janelas, os coeficientes de reflexão;

IV. Calcula-se a média dos coeficientes;

V. Calcula-se, para cada ordem, a entropia relativa da resposta impulsiva $h[n]$, tomando como referência o sinal original (envoltória do sinal de RF);

VI. A ordem que apresentar a mínima entropia será a escolhida;

VII. Encontra-se a resposta em frequência do filtro digital $1 / A(z)$; e

VIII. Analisando a resposta em frequência, a posição do ponto de máxima amplitude, dentro da janela especificada, corresponderá à frequência estimada (Figura 4). 


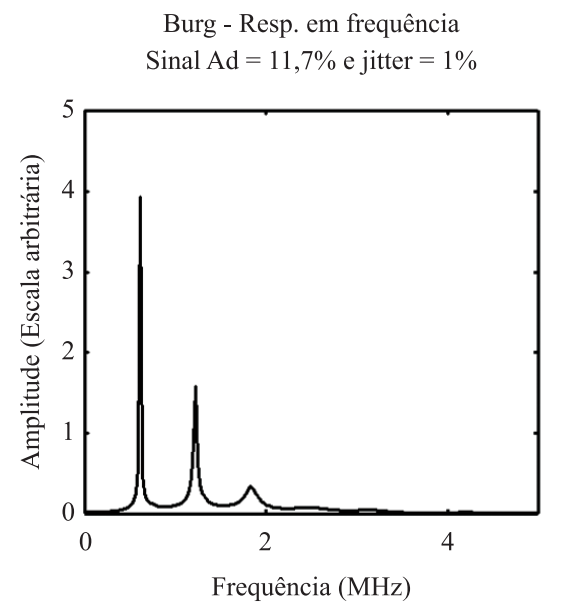

Figura 4. Exemplo da aplicação do algoritmo de BURG, com um sinal simulado de frequência central $3,5 \mathrm{MHz}$, frequência de amostragem $25 \mathrm{MHz}$ e janela de sinal de 512 amostras. Densidade espectral de potência para um sinal simulado de $\mathrm{Ad}=11,7 \%$ e jitter $=1 \%$. A FreqMSS estimada foi de $611,55 \mathrm{kHz}$.

Figure 4. Example of application of the BURG algorithm, with a simulated signal with center frequency $3.5 \mathrm{MHz}, 25 \mathrm{MHz}$ sampling frequency and signal window of 512 samples. Power spectral density for a simulated signal of $A d=11.7 \%$ and jitter $=1 \%$. The estimated FreqMSS was $611.55 \mathrm{kHz}$.

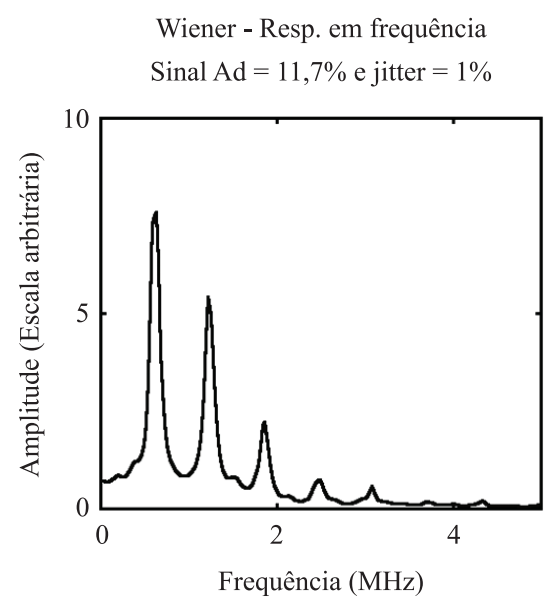

Figura 5. Exemplo da aplicação do algoritmo de WIENER, com um sinal simulado de frequência central $3,5 \mathrm{MHz}$, frequência de amostragem $25 \mathrm{MHz}$ e janela de sinal de 512 amostras. Resposta em frequência para sinal simulado de $\mathrm{Ad}=11,7 \%$ e jitter $=1 \%$. A FreqMSS estimada foi de $636,01 \mathrm{kHz}$.

Figure 5. Example of application of the WIENER algorithm, with a simulated signal with center frequency $3.5 \mathrm{MHz}, 25 \mathrm{MHz}$ sampling frequency and signal window of 512 samples. Power spectral density for a simulated signal of $A d=11.7 \%$ and jitter $=1 \%$. The estimated FreqMSS was $611.55 \mathrm{kHz}$.

\section{Método de WIENER}

A função de transferência do filtro $H(z)$ do método de WIENER (Marple, 1987) é dada pelo vetor $\left[\begin{array}{llll}1 & a[1] & \ldots & a[M]\end{array}\right]$, calculada a partir das equações, denominadas de Yule-Walker: $\left[\begin{array}{cccc}\hat{r}_{y y}[0] & \hat{r}_{y y}[-1] & \ldots & \hat{r}_{y y}[-M] \\ \hat{r}_{y y}[1] & \hat{r}_{y y}[0] & \ldots & \hat{r}_{y y}[-M+1] \\ \vdots & \vdots & \ddots & \vdots \\ \hat{r}_{y y}[M] & \hat{r}_{y y}[M-1] & \vdots & \hat{r}_{y y}[0]\end{array}\right]\left[\begin{array}{c}1 \\ a[1] \\ \vdots \\ a[M]\end{array}\right]=\left[\begin{array}{c}\sigma^{2} \\ 0 \\ \vdots \\ 0\end{array}\right]$

sendo:

$$
\begin{array}{lr}
\hat{r}_{y y}[n]=\frac{1}{N} \sum_{k=1}^{N-1-n} y[n] y[n+m] & \text { (autocorrelação); } \\
\sigma^{2}=\hat{r}_{y y}[0]+\sum_{m=1}^{N} a_{m} \hat{r_{y y}}[m] & \text { (variância do ruído). }
\end{array}
$$

Neste artigo, o método de WIENER teve as ordens determinadas pelo mínimo erro quadrático, por ter apresentado melhor índice de desempenho em relação aos resultados obtidos utilizando os métodos de Akaike (marple, 1987) ou entropia. O tamanho da janela foi definido experimentalmente, utilizando-se vários tamanhos e verificando qual obteve melhor desempenho (índice de acertos), além de verificar o número de periodicidades presentes no trecho de sinal, tendo sido encontradas 200 amostras com overlap de 100 amostras.

O algoritmo do método de WIENER é composto dos seguintes passos:

I. Define-se o tamanho da janela retangular;

II. Define-se o overlap;

III. Para cada janela, calcular o vetor de correlação da envoltória do sinal de RF;

IV. Calcula-se a média das correlações anteriores;

V. Calculam-se os coeficientes dos vetores de autocorrelação, utilizando o algoritmo de Levinson (Marple, 1987) para todas as ordens (1 até (número de amostras do trecho de sinal - tamanho da janela)/overlap) e o erro $e[n]=y[n] \cdot A(z) ;$

VI. Para cada ordem, calcula-se o erro quadrático (somatório dos erros do item anterior ao quadrado); e

VII. Para a ordem que tiver o menor erro quadrático, encontrar a resposta em frequência do filtro digital definido pelos coeficientes dos vetores de autocorrelação;

VIII. Analisando a resposta em frequência, a posição do ponto de máxima amplitude corresponderá à frequência estimada. (Figura 5)

\section{Método MUSIC}

Utilizando o estimador de frequências MUSIC (MUltiple SIgnal Classification) (Marple, 1987), a frequência referente ao espaçamento médio dos espalhadores é definida pelo ponto de máxima amplitude no espectro de MUSIC, considerando a envoltória do sinal de RF (Figura 6). 


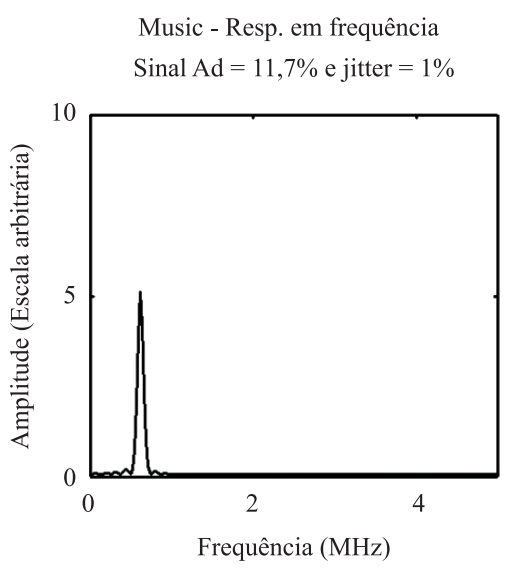

Figura 6. Exemplo da aplicação do algoritmo MUSIC, com um sinal simulado de frequência central 3,5 MHz, frequência de amostragem $25 \mathrm{MHz}$ e janela de sinal de 512 amostras. Resposta em frequência para sinal simulado de $\mathrm{Ad}=11,7 \%$ e $j i t t e r=1 \%$. A FreqMSS estimada foi de $611,55 \mathrm{kHz}$.

Figure 6. Example of application of the MUSIC algorithm, with a simulated signal with center frequency $3.5 \mathrm{MHz}, 25 \mathrm{MHz}$ sampling frequency and signal window of 512 samples. Power spectral density for a simulated signal of $\mathrm{Ad}=11.7 \%$ and jitter $=1 \%$. The estimated FreqMSS was $611.55 \mathrm{kHz}$

$$
P_{\text {MUSIC }}(f)=\frac{1}{e^{H}(f)\left(\sum_{k=M+1}^{p} a_{k} a_{k}^{H}\right) e(f)}
$$

Sendo:

$$
e(f)=\left(\begin{array}{c}
1 \\
\exp (j 2 \pi f T) \\
\vdots \\
\exp (j 2 \pi f M T)
\end{array}\right)
$$

- $T=$ metade do período limitado pela janela de análise, para $\mathrm{M}$ autovetores formadores do espaço de sinal.

\section{Pré-processamento dos sinais}

Todos os sinais foram submetidos a uma filtragem passa banda Butterworth de ordem 6, com frequências de corte relativas aos espaçamentos $0,02 \mathrm{~mm}$ $(38,5 \mathrm{MHz})$ a $3,00 \mathrm{~mm}(256,67 \mathrm{kHz})$ para os sinais reais (provenientes do phantom de fios de nylon), e de $0,10 \mathrm{~mm}(7,70 \mathrm{MHz})$ a $5,00 \mathrm{~mm}(154,00 \mathrm{kHz})$ para os sinais simulados. As frequências de corte foram calculadas para cada trecho de sinal, a partir da Equação 3.

Como a envoltória do sinal de RF possui as mesmas propriedades de espaçamento médio que o sinal de RF (Pereira e Maciel, 2001), as análises foram realizadas somente na envoltória por esta ser mais robusta para estimativa das componentes periódicas.

O sinal de envoltória foi calculado ao partir do módulo do sinal analítico, obtido via transformada de Hilbert do sinal de RF, subtraindo-se a média (para retirar o nível DC) e dividindo-se pela variância, para normalização da energia.

\section{Resultados}

\section{Sinais simulados}

A Figura 7 apresenta os resultados de percentual de acertos em função do jitter para diferentes níveis de Ad (diferentes curvas apresentadas para uma faixa de tolerância de $\pm 5 \%$ em torno do valor correto da frequência relativa ao espaçamento simulado, no caso,
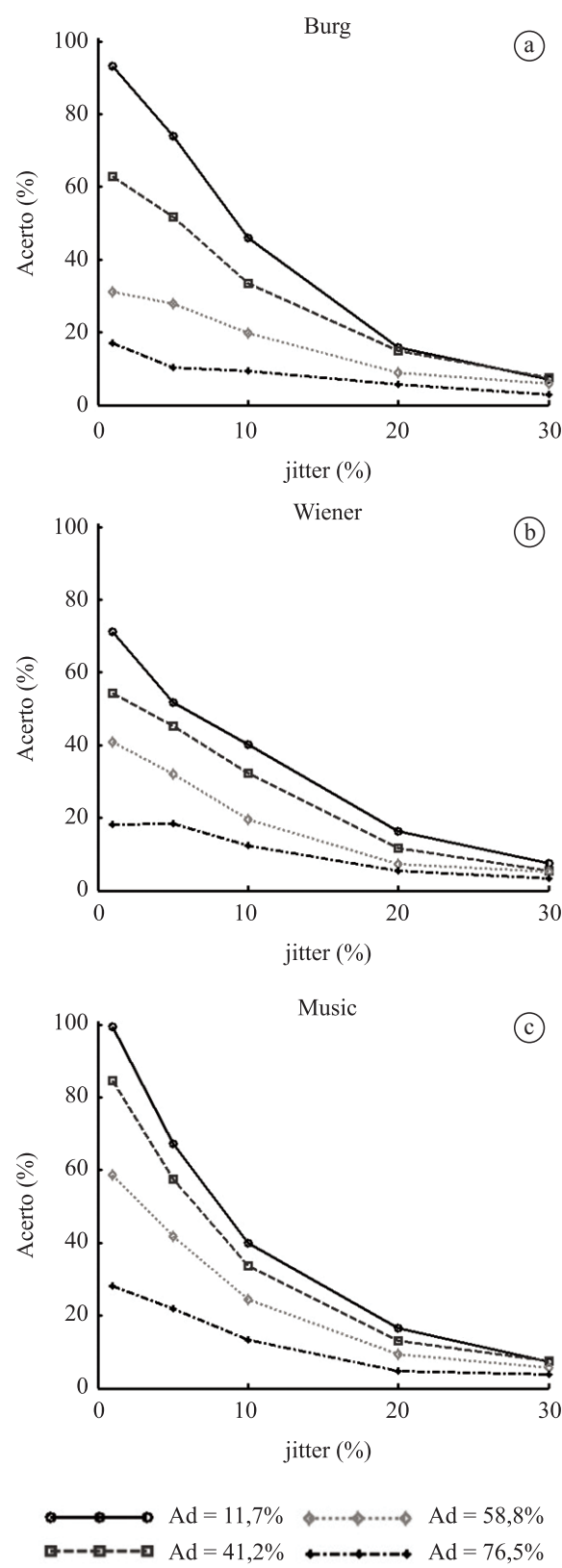

Figura 7. Resultados para sinais simulados para os métodos BURG, WIENER e MUSIC para uma faixa de tolerância de $\pm 5 \%$ em torno do valor correto $(616,00 \mathrm{MHz})$.

Figure 7. Results for simulated signals of the BURG, WIENER, and MUSIC methods for a tolerance range of $\pm 5 \%$ around the correct value $(616.00 \mathrm{MHz})$. 
Tabela 2. Resultados para sinais de fios de nylon de $0,8 \mathrm{~mm}(0,94 \mathrm{MHz})$ e $1,2 \mathrm{~mm}(0,62 \mathrm{MHz})$ para os três métodos para velocidade de US estimada igual à $1.498 \mathrm{~m} / \mathrm{s}$.

Table 2. Results for signals from phantom nylon of $0.8 \mathrm{~mm}(0.94 \mathrm{MHz})$ and $1.2 \mathrm{~mm}(0.62 \mathrm{MHz})$ for the three methods with US velocity equal to $1498 \mathrm{~m} / \mathrm{s}$.

\begin{tabular}{lcccccc}
\hline Métodos & $\begin{array}{c}\text { Espaçamento } \\
\text { entre fios (mm) }\end{array}$ & MSS (mm) & $\begin{array}{c}\text { Desvio } \\
\text { padrão }\end{array}$ & $\begin{array}{c}\mathbf{C V} \\
\mathbf{( \% )}\end{array}$ & $\begin{array}{c}\text { Faixa de } \\
\text { acerto } \\
\mathbf{\pm 5 \%}\end{array}$ & $\begin{array}{c}\text { Faixa de } \\
\text { acerto } \\
\mathbf{1 0 \%}\end{array}$ \\
\hline BURG & 0,8 & 0,61 & 0,75 & 61,48 & 22,37 & 56,58 \\
WIENER & 0,8 & 0,60 & 0,48 & 38,14 & 40,13 & 46,71 \\
MUSIC & 0,8 & 0,79 & 0,25 & 26,25 & 12,50 & 91,45 \\
BURG & 1,2 & 1,15 & 0,45 & 68,74 & 0,00 & 0,65 \\
WIENER & 1,2 & 1,10 & 0,26 & 37,92 & 0,00 & 70,39 \\
MUSIC & 1,2 & 1,17 & 0,05 & 7,51 & 0,00 & 100,00 \\
\hline
\end{tabular}

de $616,00 \mathrm{kHz}$, para uma velocidade de US no meio de $1.540 \mathrm{~m} / \mathrm{s}$ ) para os três métodos. Observa-se que os métodos tiveram um desempenho que diminuiu com o aumento dos níveis de jitter ou Ad, como esperado.

\section{Phantoms de fios de nylon}

Após o estudo com sinais simulados, realizou-se uma análise com sinais de phantom de fios de nylon, com espaçamentos de $0,8 \mathrm{~mm}(0,94 \mathrm{MHz})$ e $1,2 \mathrm{~mm}$ $(0,62 \mathrm{MHz})$ cujos resultados estão apresentados na Tabela 2.

\section{Discussão}

Para sinais simulados, o método MUSIC tende a ter um desempenho melhor para os dois casos mais favoráveis no qual Ad e jitter são menores, ou seja, $\mathrm{Ad}=11,7 \%$ e $41,2 \%$, até um valor de jitter de $10 \%$. O método de BURG, por sua vez, tem desempenho melhor que o de WIENER para esta mesma faixa. A partir deste ponto (jitter $=10 \%)$, o desempenho diminui rapidamente, o que sugere que a periodicidade não é mais evidente (não se pode dizer que há uma periodicidade). Importante ressaltar que, nos sinais simulados, a característica dominante é o emprego de periodicidades com maior ou menor quantidade de ruído associado. Não há grandes alvos refletores isolados ou estruturas altamente atenuadoras que gerem sinais mais complexos. Isso permite que se estude a influência da variação independente do jitter e do Ad, na estimativa da periodicidade com cada método. Comparando com a literatura, os três métodos apresentaram resultados equivalentes a Simon (1997) e Pereira e Maciel (2001), como na transformação quadrática do sinal (Simon, 1997) e no uso do SSA (Singular Spectrum Analysis) (Pereira e Maciel, 2001), mas com a vantagem de ser automático.

Utilizando os sinais provenientes de phantom de fios de nylon, para a faixa de acerto de $\pm 5 \%$, todos os métodos tiveram um erro maior que $50 \%$. Entretanto, considerando-se a faixa de acerto de $\pm 10 \%$ os métodos
WIENER e MUSIC apresentaram melhor índice para fios espaçados de $1,2 \mathrm{~mm}$.

É válido ressaltar que todos os métodos apresentaram a média da frequência estimada (Tabela 2) acima de 0,94 MHz (correspondente a uma periodicidade inferior a $0,8 \mathrm{~mm}$ ) e acima de $0,62 \mathrm{MHz}$ (correspondente a uma periodicidade inferior a 1,2 mm) como era esperado, já que o feixe de US incide inclinado ao plano formado pelos fios de nylon, o que levaria a uma estimativa menor que o espaçamento real.

Para estes phantoms, o método MUSIC apresentou os melhores resultados, considerando média e desvio-padrão. Importante salientar que os sinais dos phantoms de fios de nylon são sinais reais simples, cuja periodicidade pode ser identificada visualmente, porém, percebe-se uma variação importante de amplitudes entre os ecos, sendo, portanto, um teste inicial da capacidade dos métodos de estimar o MSS, levando em conta essa característica.

Como o método é automático, foi utilizada uma janela de análise ampla que pudesse estimar qualquer espaçamento, entretanto, isto torna o método suscetível à influência de eventuais grandes refletores, gerando estimativas de espaçamento entre estes e não o desejado.

Utilizando sinais do phantom de fios de nylon, somente o método de MUSIC apresentou resultados equivalentes aos da literatura com este meio Pereira e Maciel (2001), isso pode ser devido ao fato do SSA também separar os espaços de sinais periódicos com sinais aperiódicos.

\section{Conclusão}

Este trabalho avalia três métodos (BURG, WIENER e MUSIC), apresentando como originalidade a determinação da ordem de cada método para cada estimativa de espaçamento, de forma automática (sem ser necessária a intervenção do operador). O método 
MUSIC apresentou melhor resultado global o que é uma indicação de que deveria ser melhor investigado com vistas a uma futura utilização clínica.

\section{Referências}

Amin AN, Ebbini ES, Georgiou TT. Noninvasive estimation of tissue temperature via high-resolution spectral analysis techniques. IEEE Transactions on Biomedical Engineering. 2005; 52(2):221-8.

Bridal SL, Fornès P, Bruneval P, Berger G. Correlation of ultrasonic attenuation ( 30 to $50 \mathrm{MHz}$ ) and constituents of atherosclerotic plaque. Ultrasound in Medicine \& Biology. 1997; 23:691-703. http://dx.doi.org/10.1016/ S0301-5629(97)00072-0

Calas MJG, Almeida RMVR, Gutfilen B, Pereira WCA. Intraobserver interpretation of breast ultrasonography following the BI-RADS classification. European Journal of Radiology. 2010; 74(3):525-8. PMid:19423260. http:// dx.doi.org/10.1016/j.ejrad.2009.04.015

Cover TM, Thomas JA. Elements of information theory. New Jersey: Wiley-Interscience; 1991.

Fellingham L, Sommer F. Ultrasonic characterization of tissue structure in the in vivo human liver and spleen. IEEE Transactions on Sonics and Ultrasonics. 1984; SU-31:418-28.

Huang K, Ta D, Wang W, Le LH. Simplified inverse filter tracking algorithm for estimating the mean trabecular bone spacing. IEEE Transactions on Ultrasonics, Ferroelectrics, and Frequency Control. 2008; 55(7):1453-64.

Insana M, Hall TJ, Fishback JL. Identifying acoustic scattering sources in normal renal parenchyma from the anisotropy in acoustic properties. Ultrasound in Medicine \& Biology. 1991; 17:613-26. http://dx.doi. org/10.1016/0301-5629(91)90032-R

Kauati AT, Campos M, Pereira WCA. Cálculo de entropia e análise de espectro singular em sinais senoidais e de RF ultrassônicos simulados. In: XVII Congresso Brasileiro de Engenharia Biomédica: Anais do XVII Congresso Brasileiro de Engenharia Biomédica; 11-13 Set 2000; Florianópolis, Santa Catarina. Florianópolis; 2000. p. 1290-5.

Landini L, Verrazzani L. Spectral characterization of tissue microstructure by ultrasound: a stochastic approach. IEEE Transactions on Ultrasonics, Ferroelectrics, and Frequency
Control.1990; 37:448-56. PMid:18285062. http://dx.doi. org/10.1109/58.105251

Machado CB, Pereira WCA, Meziri M, Laugier P. Characterization of in vitro healthy and pathological human liver tissue periodicity using backscattered ultrasound signals, Ultrasound in Medicine \& Biology. 2006; 32(5):649-57. PMid:18285062. http://dx.doi.org/10.1016/j. ultrasmedbio.2006.01.009

Marple SL. Digital spectral analysis: with applications. New Jersey: Prentice-Hall; 1987.

Narayanan VM, Molthen RC, Shankar PM, Vergara LJ, Reid M. Studies on ultrasonic scattering from quasi-periodic structures. IEEE Transactions on Ultrasonics, Ferroelectrics, and Frequency Control. 1997; 44:114-24. PMid:18244109. http://dx.doi.org/10.1109/58.585205

Ossant F, Lebertre M, Pourcelot L, Patat F. Ultrasonic characterization of maturation of fetal lung microstructure: an animal study. Ultrasound in Medicine \& Biology. 2001; 27:157-69. PMid:18244109. http://dx.doi. org/10.1016/S0301-5629(00)00335-5

Pereira WCA, Abdelwahab A, Lori BS, Laugier P. Singular Spectrum Analysis Applied to $20 \mathrm{MHz}$ Backscattered Ultrasound Signals From Periodic And Quasi-Periodic Phantoms. Acoustical Imaging. 2002; 26:239-46. http:// dx.doi.org/10.1007/978-1-4419-8606-1_31

Pereira WCA, Maciel CD. Performance of ultrasound echo decomposition using singular spectrum analysis. Ultrasound in Medicine \& Biology. 2001; 27(9):1231-8. PMid:18285062. http://dx.doi.org/10.1016/S0301-5629(01)00423-9

Simon C, Shen J, Seip R, Ebbini ES. A Robust and computationally efficient algorithm for mean scatterer spacing estimation. IEEE Transactions on Ultrasonics, Ferroelectrics, and Frequency Control. 1997; 44(4): 882-94. http://dx.doi.org/10.1109/58.655203

Varghese T, Donohue KD. Mean scatterer spacing estimate with spectral correlation. Journal of Acoustical Society of America. 1994; 96:3504-15. PMid:7814765. http://dx.doi. org/10.1121/1.410611

Wear KA, Wagner RF, Insana MF, Hall TJ. Application of autoregressive spectral analysis to cepstral estimation of mean scatterer spacing. IEEE Transactions on Ultrasonics, Ferroelectrics, and Frequency Control. 1993; 40(1):50-8. PMid:18263156. http://dx.doi.org/10.1109/58.184998

\section{Autores}

\section{Adriana Kauati*}

Engenharia Elétrica, Centro de Engenharias e Ciências Exatas - CECE, Universidade Estadual do Oeste do Paraná - UNIOESTE, Av. Tarquinio Joslin dos Santos, 1300, Polo Universitário, CEP 85870-650, Foz do Iguaçu, PR, Brasil.
}

\section{Wagner Coelho de Albuquerque Pereira}

Programa de Engenharia Biomédica, Instituto Alberto Luiz Coimbra de Pós-Graduação e Pesquisa de Engenharia - COPPE, Universidade Federal do Rio de Janeiro - UFRJ, Rio de Janeiro, RJ, Brasil.

\section{Marcello Luiz Rodrigues de Campos}

Programa de Engenharia Elétrica, Instituto Alberto Luiz Coimbra de Pós-Graduação e Pesquisa de Engenharia - COPPE, Universidade Federal do Rio de Janeiro - UFRJ, Rio de Janeiro, RJ, Brasil. 